\title{
Microscopic entropy of trapping horizon
}

\author{
Mikhail Z. Iofa ${ }^{1}$ \\ Skobeltsyn Institute of Nuclear Physics \\ Moscow State University \\ Moscow 119991, Russia
}

\begin{abstract}
In the Carlip-Majhi-Padmanabhan approach, we calculate microscopic entropy of trapping (apparent) horizon of the FRW metric. We solve Killing equations for $t, r$ part of the metric without fixing a piori the form of the scaling factor $a(t)$ which is determined from the requirement of consistency of Killing equations. Further restrictions on the form of the Killing vector follow from the requirement that Killing vector is null at the trapping horizon at all $t$. Applying the technique used to calculate microscopic entropy of Killing horizons, we calculate microscopic entropy of trapping horizon. Using the explicit form of Killing vector, we verify that identities used in calculation of the central term of Virasoro algebra for Killing horizons of black holes are valid in the present case.
\end{abstract}

\section{Introduction}

Since the discovery of thermodynamic nature of black holes [1,2], calculation of entropy of black holes has attracted considerable attention. On the one hand thermodynamic properties of static event horizons of black holes were studied and their "geometric entropy" was calculated. Parallel to the study of black hole horizons and their thermodynamic properties also cosmological horizons and their thermodynamics were intensively studied $[3,4,5,6,7,8]$. In the course of these investigations it was realized that in cosmological cases more relevant is not the event horizon, but apparent and trapping horizons $[4,9,10,11,12]$.

On the other hand, much effort was put to calculation of "microscopic entropy". This approach initiated in [13] originally in $2+1$ gravity was subsequently developed in many papers. The approach is based on the result [14] that in $2+1$ gravity with negative cosmological constant the black hole possesses asymptotic symmetry consisting of two copies of the Virasoro algebra. Substituting the central charge of the Virasoro algebra in the Cardy formula, it was possible to calculate the density of states and to obtain microscopic entropy of black hole which appeared to be equal to the geometric one. Next, the approach based on the use of the asymptotic symmetry, was generalized to $3+1$ theory [15] and applied to the Kerr black hole [16].

Another method to calculate microscopic entropy of black holes was developed in papers of Carlip [17], who showed that symmetries of near-horizon geometry imply the algebra of constraints with central extension which contains the Virasoro sub-algebra with central charge. The method was applied to 2D, 3D and 4D black holes [17, 20]. In particular, in this approach were obtained the results of [16] for Kerr/CFT [19]. Carlip's approach was refined in [18], and [21], with the use of new canonical hamiltonian which satisfies the variation principle in presence of boundaries. Near-horizon geometry was investigated in [22] The above results were based on the on-shell constructions (see review [21]).

Further development of the approach based on the use of the algebra of diffeomorphisms in the near-horizon region was performed in papers [24], where an off-shell construction of the algebra of

\footnotetext{
${ }^{1}$ e-mail: iofa@theory.sinp.msu.ru
} 
constraints was developed. The off-shell Noether current was used to calculate the central charge of the algebra of diffeomorphisms and to obtain the Wald entropy [25]. The method provides a straightforward derivation of the Virasoro algebra and calculation of the central term.

The above results which refer to local Killing horizons of stationary black holes, defined as a surface at which Killing vector is null, were generalized to a more general case of isolated horizons [26], which are generalization of Killing horizon of black hole, and which include non-stationary geometries. Imposing boundary conditions on the metric in the near-horizon region, a set of diffeomorphisms was constructed with the corresponding Noether charges forming the Virasoro algebra.

In the study of metrics with dynamically evolving non-stationary horizons rather than stationary black holes, definition of the boundary of a stationary black hole as a hypersurface at which Killing vector is null was changed to definition of the boundary as a trapping horizon which is the surface where Kodama vector is null [27, 28, 29]. Thermodynamic of evolving horizons was studied by substitution of Kodama vector and trapping horizon for Killing vector and Killing horizon.

In this paper we study possibility to calculate microscopic entropy of trapping horizon of sphericallysymmetric metrics

$$
d s^{2}=-d t^{2}+a^{2}(t) b^{2}(r) d r^{2}+R^{2}(r, t) d \Omega^{2}
$$

by reducing the problem to calculation through the null Killing vector. For this class of metrics condition that Kodama vector $K^{i}=(1 / \sqrt{-g}) \epsilon^{i j} \partial_{j} R$ is null can be alternatively written as $g^{i k} \partial_{i} R \partial_{k} R=0$ $[27,28,30]$. To perform a calculation of microscopic entropy of the trapping horizon, we look for a Killing vector of the metric $-d t^{2}+a^{2}(t) b^{2}(r) d r^{2}$. Solving Killing equations we do not fix the scaling factor $a(t)$ which form is restricted by consistency conditions of the system of Killing equations. Further restrictions on the components of the Killing vector are obtained, by demanding that Killing vector is null at the trapping horizon for all $t$.

Because by construction Killing vector is null at the trapping horizon, Killing and trapping horizons coincide, and we can apply the well-developed methods of calculation of microscopic entropy of local Killing horizons [17, 19, 20, 22, 24]. We use the off-shell approach of [24]. We find that Killing vector null at the trapping (apparent) horizon exists in the case of the FRW metric with $b=1$ and $R^{2}=a^{2}(t) r^{2}$. We prove that relations derived in [17] and [24] used in calculations of the central term of the Virasoro algebra are valid in the present case. We calculate microscopic entropy of the trapping horizon which coincides with the result of thermodynamic calculations (reviews $[28,31,8]$ ).

We also consider the FRW metric with $b^{2}(r)=\left(1 \pm r^{2}\right)^{-1}$ and find that in these cases Killing equations do not admit null Killing vectors on trapping horizon.

\section{Killing vectors on trapping horizon}

We consider a spatially homogeneous isotropic space-time with the metric

$$
d s^{2}=g_{i j} d x^{i} d x^{j}+R^{2}(x) d \Omega^{2}=-d t^{2}+a^{2}(t) \frac{d r^{2}}{1-k r^{2}}+a^{2}(t) r^{2} d \Omega^{2},
$$

where $k= \pm 1,0$. We begin with the metric with $k=0$. Killing equations $L_{\chi} g_{i j}=0$ have the form

$$
\begin{aligned}
& \chi^{c} \partial_{c} g_{t t}+2 \partial_{t} \chi^{c} g_{c t}=0, \\
& \chi^{c} \partial_{c} g_{r r}+2 \partial_{r} \chi^{c} g_{c r}=0, \\
& \chi^{c} \partial_{c} g_{r t}+\partial_{t} \chi^{c} g_{c r}+\partial_{r} \chi^{c} g_{c t}=0 .
\end{aligned}
$$


Substituting explicit expressions for the metric, we reduce Killing equations to a system

$$
\begin{aligned}
& \dot{\chi}^{t}=0, \\
& 2\left(\chi^{r}\right)^{\prime} a^{2}+\chi^{t}\left(\dot{a^{2}}\right)=0, \\
& -\left(\chi^{t}\right)^{\prime}+\dot{\chi}^{r} a^{2}=0,
\end{aligned}
$$

where dot and prime are derivatives over $t$ and $r$. Integrating the system, we obtain

$$
\begin{aligned}
& \chi^{t}=f(r), \\
& \chi^{r}=-\frac{\dot{a}}{a} \int^{r} f\left(r^{\prime}\right) d r^{\prime}+\varphi(t), \\
& \chi^{r}=f^{\prime}(r) \int^{t} \frac{d t^{\prime}}{a^{2}\left(t^{\prime}\right)}+\psi(r) .
\end{aligned}
$$

where $f, \varphi$ and $\psi$ are arbitrary functions. Taking derivatives of (9) and (10) over $r$ and $t$ and equating the resulting expressions, we obtain

$$
f^{\prime \prime}(r)+\left(\ddot{a}(t) a(t)-\dot{a}^{2}(t)\right) f(r)=0
$$

For consistency of Eq. (11) it is necessary that $\ddot{a} a(t)-\dot{a}^{2}(t)=$ const.

\subsection{The case $\ddot{a} a-\dot{a}^{2}=0$}

First we consider the case const $=0$. Equation $\ddot{a} a-\dot{a}^{2}=0$ has solution

$$
a(t)=C_{1} e^{-H t}
$$

where $H$ and $C_{1}$ are integration constants. From (11) we have

$$
f(r)=k_{1}+k_{2} r
$$

Eqs. (8) - (10) yield

$$
\begin{aligned}
& \chi^{t}=k_{1}+k_{2} r \\
& \chi^{r}=H\left(k_{1} r+k_{2} \frac{r^{2}}{2}+C^{\prime}\right)+\varphi(t) \\
& \chi^{r}=k_{2}\left(\frac{e^{2 H t}}{2 H C_{1}^{2}}+C^{\prime \prime}\right)+\psi(r) .
\end{aligned}
$$

Equating expressions (15) and (16) and noting that dependence on $r$ and $t$ is separated, we have

$$
\varphi(t)=\frac{k_{2}}{2 H a^{2}(t)}+C_{2}, \quad \psi(r)=H\left(k_{1} r+k_{2} \frac{r^{2}}{2}\right)+C_{3},
$$

where $H C^{\prime}+C_{2}=k_{2} C^{\prime \prime}+C_{3} \equiv C$. Substituting these relations in (15) and (16), we obtain

$$
\chi^{r}=H\left(k_{1} r+k_{2} \frac{r^{2}}{2}\right)+\frac{k_{2}}{2 H a^{2}}+C
$$


Collecting (14) and (17), we have

$$
\chi^{a}=\left(k_{1}+k_{2} r ; \quad H\left(k_{1} r+k_{2} \frac{r^{2}}{2}\right)+\frac{k_{2}}{2 H a^{2}}+C\right)
$$

and

$$
\chi^{2}=-\left(k_{1}+k_{2} r\right)^{2}+a^{2} H^{2}\left(k_{1} r+k_{2} \frac{r^{2}}{2}+\frac{k_{2}}{2 H^{2} a^{2}}+C\right)^{2} .
$$

Dynamical trapping horizon is found by solving the equation

$$
g^{i k} \partial_{i} R \partial_{k} R=0
$$

Eq. (20) yields

$$
r_{h}=\frac{1}{|\dot{a}(t)|}=\frac{1}{H a(t)} .
$$

It is seen that at the trapping horizon the Killing vector (18) with $C=0$

$$
\left.\chi^{a}\right|_{h o r}=\left(k_{1}+\frac{k_{2}}{H a} ; \frac{1}{a}\left(k_{1}+\frac{k_{2}}{H a}\right)\right)
$$

is null.

There exist also a solution with $f(r)=0, \quad \chi^{a}=\left(\chi^{t}, \chi^{r}\right)=(0,1)$. However, the norm of this Killing vector is $\chi^{2}=a^{2}(t)$ and does not vanish on horizon.

\subsection{The case $\ddot{a} a-\dot{a}^{2}= \pm m^{2}$}

Let us consider the case const $=-m^{2}$. Equation $\ddot{a} a-\dot{a}^{2}=-m^{2}$ has solution

$$
a(t)=\frac{m}{H} \sinh (H t+p)
$$

where $H$ and $p$ are arbitrary. Solution of the Eq. (11), $f^{\prime \prime}-m^{2} f=0$, is

$$
f(r)=C_{1} e^{m r}+C_{2} e^{-m r}
$$

From Eqs.(3) and (4) we obtain

$$
\chi^{r}=-\frac{H}{m^{2}} f^{\prime}(r)\left(\operatorname{coth}(H t+p)+C^{\prime}\right)+\psi(r)
$$

and

$$
\chi^{r}=-\frac{H}{m^{2}} \operatorname{coth}(H t+p)\left(f^{\prime}(r)+C^{\prime \prime}\right)+\varphi(t),
$$

where $\psi$ and $\varphi$ are arbitrary functions. Equating (25) and (26), we obtain

$$
-\frac{H}{m^{2}} f^{\prime}(r) C^{\prime}+\psi(r)=-\frac{H}{m^{2}} \operatorname{coth}(H t+p) C^{\prime \prime}+\varphi(t)=C
$$

where

$$
\psi(r)=C+\frac{H}{m^{2}} f^{\prime}(r) C^{\prime} ; \quad \varphi(t)=C+\frac{H}{m^{2}} \operatorname{coth}(H t+p) C^{\prime \prime}
$$


. Substituting (28), we find the Killing vector

$$
\chi^{a}=\left(f(r) ;-\frac{H}{m^{2}} f^{\prime}(r) \operatorname{coth}(H t+p)+C\right) .
$$

Horizon is located at

$$
r_{h}^{-1}=m \cosh (H t+p) .
$$

It is seen that condition $\chi_{h}^{2}=0$ is not satisfied for all $t^{2}$.

The case $\ddot{a} a-\dot{a}^{2}=m^{2}$ is obtained by substitution $m \rightarrow i m$ and leads to the same conclusion as above.

\subsection{Killing vectors for the metric with $k= \pm 1$}

To be precise, we consider Killing equations for the metric with $k=-1$

$$
d s^{2}=-d t^{2}+d r^{2} \frac{a^{2}(t)}{1+r^{2}}+a^{2}(t) r^{2} d \Omega^{2} .
$$

Killing equations are

$$
\begin{aligned}
& \dot{\chi}^{t}=0, \quad \chi^{t} \Rightarrow f(r) \\
& \chi^{r} a^{2} \frac{-2 r}{\left(1+r^{2}\right)^{2}}+\chi^{t} \frac{\left(\dot{a^{2}}\right)}{1+r^{2}}+2\left(\chi^{r}\right)^{\prime} \frac{a^{2}}{1+r^{2}}=0 \\
& -\left(\chi^{t}\right)^{\prime}+\dot{\chi}^{r} \frac{a^{2}}{1+r^{2}}=0
\end{aligned}
$$

or

$$
\begin{aligned}
& \left(\chi^{r}\right)^{\prime}+\frac{\dot{a}}{a} f-\chi^{r} \frac{r}{1+r^{2}}=0 \\
& \dot{\chi}^{r}=f^{\prime} \frac{1+r^{2}}{a^{2}}
\end{aligned}
$$

Taking derivative of Eqs.(34) and (35) over $t$ and $r$ and equating the resulting expressions for $\dot{\chi}^{r^{\prime}}$, we obtain consistency equation

$$
\left[f^{\prime}(r)\left(1+r^{2}\right)\right]^{\prime}-f^{\prime}(r) r+\left[\ddot{a}(t) a(t)-\dot{a}(t)^{2}\right] f(r)=0 .
$$

In the case $\ddot{a} a-\dot{a}^{2}=0$ we obtain $a(t)=C_{1} \exp \{-H t\}$ and

$$
f(r)=k_{1}+k_{2} \ln \left[r+\sqrt{1+r^{2}}\right]
$$

where $k_{1,2}$ are integration constants. Substituting $f(r)$ and $a(t)$ in Eqs. (32) and (33) and performing integration, we obtain solutions of these equations

$$
\begin{aligned}
& \chi^{r}=\sqrt{1+r^{2}}\left(\varphi(t)+H \int d r \frac{f(r)}{\sqrt{1+r^{2}}}\right)=\sqrt{1+r^{2}}\left(\varphi(t)+\frac{f^{2}(r) H}{2 k_{2}}+C^{\prime}\right) \\
& \chi^{r}=k_{2} \sqrt{1+r^{2}} \int \frac{d t}{a^{2}(t)}+\psi(r)=\sqrt{1+r^{2}}\left(\frac{k_{2}}{2 H a^{2}(t)}+C^{\prime \prime}\right)+\psi(r),
\end{aligned}
$$

\footnotetext{
${ }^{2}$ For example, taking in (24) $C_{2}=0$ from condition $\chi_{h}^{2}=0$ we obtain $\pm 1=\frac{H}{m} \operatorname{coth}(H t+p)+C$.
} 
where $\varphi(t)$ and $\psi(r)$ are arbitrary functions. Equating expressions for $\chi^{r} / \sqrt{1+r^{2}}$ which follow from (38) and (39) and noting that dependence on $r$ and $t$ is separated, we obtain

$$
C^{\prime}+\varphi(t)-\frac{k_{2}}{2 H a^{2}(t)}=C^{\prime \prime}+\frac{\psi(r)}{\sqrt{1+r^{2}}}-\frac{f^{2}(r) H}{2 k_{2}}=C
$$

Killing vector is

$$
\chi^{a}=\left(f ; \sqrt{1+r^{2}}\left(\frac{H}{2 k_{2}} f^{2}+\frac{k_{2}}{2 H a^{2}}+C\right)\right) .
$$

Trapping horizon is located at

$$
r_{h}^{2}=\left(\dot{a}^{2}-1\right)^{-1}
$$

Square of the Killing vector is

$$
\chi^{2}=-f^{2}+a^{2}\left(\frac{H}{2 k_{2}} f^{2}+\frac{k_{2}}{2 H a^{2}}+C\right)^{2} .
$$

Supposing that $\chi^{2}$ vanishes on horizon, we have

$$
f\left(r_{h}\right)= \pm \frac{k_{2}}{H a}+\tilde{C}
$$

or, using (37),

$$
k_{1}+\frac{k_{2}}{2} \ln \frac{H a+1}{H a-1}= \pm \frac{k_{2}}{H a}+\tilde{C} .
$$

Relation (44) is not valid for all $t$, and we conclude that $\chi^{2}$ does not vanish on the horizon for all $t$.

In the case $\ddot{a} a-\dot{a}^{2}=-m^{2}$ we solution for $a(t)$ and $f(r)$ is

$$
a(t)=\frac{m}{H} \sinh (H t+p), \quad f(r)=k_{1} p^{m}+k_{2} p^{-m},
$$

where

$$
p=r+\sqrt{1+r^{2}} .
$$

Trapping horizon is at

$$
r_{h}^{2}=\left(m^{2} \operatorname{coth}^{2}(H t+p)-1\right)^{-1}
$$

Killing vector has the following structure

$$
\chi^{a}=\left(k_{1} p^{m}+k_{2} p^{-m} ;-\frac{H}{m} \sqrt{1+r^{2}}\left(k_{1} p^{m}-k_{2} p^{-m}\right) \operatorname{coth}(H t+p)\right) .
$$

Assuming that the square of the Killing vector is null at the trapping horizon, we obtain a relation

$$
\sqrt{m^{2} \operatorname{coth}^{2}(H t+p)-1}=m \operatorname{coth}^{2}(H t+p)
$$

which is not valid for all $t$.

To conclude, the metrics (1) with $k \neq 0$ do not admit Killing vectors null at the trapping horizon. 


\section{Local Killing horizon}

In this section we introduce objects used to construct the Virasoro algebra. The surface gravity is defined as

$$
\kappa^{2}=-\left.\frac{1}{2} \nabla^{a} \chi^{b} \nabla_{a} \chi_{b}\right|_{h o r}
$$

For the Killing vector (17) we obtain ${ }^{3}$

$$
\kappa^{2}=H^{2} k_{1}^{2}
$$

Next, we define the vector $\rho_{a}$

$$
\rho_{a}=-\frac{1}{2 \kappa} \nabla_{a} \chi^{2}
$$

which explicitly is

$$
\rho_{a}=\frac{1}{k_{1}}\left((a H F)^{2}-\left(\frac{k_{2}}{2 H a}\right)^{2} ; a F^{\prime}\left(\frac{k_{2}}{2 H a}-F H a\right)\right) .
$$

It is verified explicitly that $\chi^{a} \rho_{a}=0{ }^{4}$. On the horizon we have

$$
\rho_{a, h o r}=\left(k_{1}+\frac{k_{2}}{H a} ;-a\left(k_{1}+\frac{k_{2}}{H a}\right)\right) \text {. }
$$

Sufficient condition that surface variations keep position of horizon fixed is

$$
\frac{\chi^{a} \chi^{b} \delta g_{a b}}{\chi^{2}} \rightarrow 0
$$

as $\chi^{2} \rightarrow 0$. Carlip considered diffeomorphisms of the form

$$
\xi^{a}=T \chi^{a}+R \rho^{a}
$$

For these diffeomorphisms one has

$$
\delta \chi^{2}=\chi^{a} \chi^{b} \delta_{\xi} g_{a b}=2 \chi^{2} D T-2 \kappa \rho^{2} R,
$$

where $D=\chi^{a} \nabla_{a}$. To form a closed algebra of diffeomorphisms near horizon the functions $T$ and $R$ are constrained by additional conditions $[17,24]^{5}$.

$$
\rho^{a} \nabla_{a} T=0, \quad R=-\frac{\chi^{2}}{\kappa \rho^{2}} D T, \quad \nabla_{a} T=\frac{\chi_{a}}{\chi^{2}} D T
$$

In the near-horizon region the first condition (56) reduces to

$$
\left(k_{1}+\frac{k_{2}}{H a}\right)\left(\partial_{t} T(r, t)+\frac{1}{a(t)} \partial_{r} T(r, t)\right)=0
$$

with solution $T=T(r-1 / a H)$. The second condition (56) in the hear-horizon region is

$$
R=-\frac{\chi^{2}}{\kappa \rho^{2}}\left(k_{1}+\frac{k_{2}}{H a}\right)\left(\partial_{t} T+\frac{1}{a} \partial_{r} T\right) .
$$

Because of (57), $R$ is zero at the horizon. In Appendix it is shown that the ratio $\chi^{2} / \rho^{2}$ is finite at the horizon.

\footnotetext{
${ }^{3}$ Because Killing vector $\chi^{a}$ is defined up to a constant factor, we can set $k_{1}=1$. In this case the surface gravity (49) coincides with that defined in [28].

${ }^{4}$ It is also valid on general grounds since $\chi^{a}$ is a Killing vector.

${ }^{5}$ It seems that in the off-shell approach condition $\int d^{2} x \sqrt{h} D^{3} T=0$ is unnecessary due to cancellations.
} 


\section{Algebra Virasoro and Cardy formula}

In this section we briefly consider some steps of the Carlip-Majhi-Padmanabhan approach leading to the Virasoro algebra. The formulas which were derived in $[17,24]$ using only properties of Killing vectors require no further justification, validity of those which were obtained relying on some properties of static horizons are discussed in Appendix. Below we follow notations of [17, 24].

The current associated with the diffeomorphism $\xi^{a}(54)$ is given by

$$
J^{a}=\frac{1}{8 \pi G}\left(\nabla^{d} \nabla^{a} \xi_{d}-\nabla^{2} \xi^{a}\right)
$$

In the vicinity of horizon the current takes the form

$$
J^{a}=-\frac{1}{8 \pi G} \frac{\rho^{a}}{\chi^{2}}\left(2 \kappa D T-\frac{1}{\kappa} D^{3} T\right)+O\left(\chi^{2}\right)
$$

The Noether charge is corresponding to diffeomorphisn $\xi$ is

$$
\begin{aligned}
Q(\xi)=\quad & \int d \Sigma_{a} \sqrt{g} J^{a}=\int d \Sigma_{a b} \sqrt{h} J^{a} \xi^{b} \\
= & -\frac{1}{32 \pi G} \int d^{2} x \sqrt{h}\left|\frac{\rho}{\chi}\right|\left(g^{a c} g^{b d}-g^{a d} g^{b c}\right) \mu_{a b} \mu_{c d}\left(2 \kappa D T-\frac{1}{\kappa} D^{3} T\right)
\end{aligned}
$$

Where $d \Sigma_{a b}=d^{2} x \mu_{a b}$ is the surface element on the Killing horizon. Constructing

$$
d \Sigma_{a b} J^{b}\left(\xi_{1}\right) \xi_{2}^{a}=\frac{d^{2} x}{32 \pi G}\left|\frac{\rho}{\chi}\right|\left(g^{a c} g^{b d}-g^{a d} g^{b c}\right) \mu_{a b} \mu_{c d}\left(2 \kappa D T_{1}-\frac{1}{\kappa} D^{3} T_{1}\right) T_{2}
$$

one obtains commutator $\left[Q_{1}, Q_{2}\right]=Q\left(\left\{\xi_{1}, \xi_{2}\right\}\right)+K\left(\xi_{1}, \xi_{2}\right)$ where the central term $K\left(\xi_{1}, \xi_{2}\right)$ in the near-horizon limit is

$$
K\left(\xi_{1}, \xi_{2}\right)=-\frac{1}{32 \pi G} \int d^{2} x \sqrt{h}\left(g^{a c} g^{b d}-g^{a d} g^{b c}\right) \mu_{a b} \mu_{c d} \frac{1}{\kappa}\left(D T_{1} D^{2} T_{2}-D T_{2} D^{2} T_{1}\right) .
$$

Next, one defines decomposition of $T_{1,2}$ in the basis

$$
\left\{T_{n}=\frac{1}{\kappa} e^{i n \kappa v+f\left(\tau^{i}\right)}\right\}
$$

Here $f\left(\tau^{i}\right)$ is a function of other coordinates regular on horizon and $v$ is defined as $\chi^{a} \nabla_{a} v=1^{6}$. It follows that

$$
\left\{T_{m}, T_{n}\right\}=T_{m} D T_{n}-T_{n} D T_{m}=-i(m-n) T_{m+n}
$$

Using (60)-(61) it is shown that

$$
i\left[Q\left(\xi_{m}\right), Q\left(\xi_{n}\right)\right]=(m-n) Q\left(\xi_{m+n}\right)+m^{3} \frac{A}{8 \pi G} \delta_{m+n, 0}
$$

where $A$ is the horizon area.

Defining the central charge and zero mode eigenvalue, and substituting them in the Cardy formula, one obtains the entropy equal to the geometric one, i.e. $S=A / 4 G$.

\footnotetext{
${ }^{6}$ In the near-horizon region $v=v(r-1 / a(t) H)$.
} 


\section{Conclusions}

In this paper we calculated microscopic entropy of trapping horizon of the FRW metric by looking for a null Killing vector at the trapping horizon of the 2D $r, t$ part of the metric. Having a null Killing vector, we can apply the well-developed technique of calculation of microscopic entropy of null surfaces. Killing equations are solved without a priori fixing the form of the scaling factor $a(t)$, which is determined from consistency condition of Killing equations $\ddot{a} a-\dot{a}^{2}=$ const. We have found that in the case const $=0$ the FRW metric (1) with $k=0$ has Killing vector null at the trapping horizon. In this case the metric is $\mathrm{dS}$ metric. The metrics (1) with $k= \pm 1$ do not admit Killing vectors null at the trapping horizon.

The metric (1) with $k=0$ is De Sitter metric in the "flat slicing". In static coordinates De Sitter metric is

$$
d s^{2}=-\left(1-\frac{r^{2}}{H^{2}}\right) d t^{2}+\left(1-\frac{r^{2}}{H^{2}}\right)^{-1} d r^{2}+r^{2} d \Omega^{2}
$$

In this form the metric has static event horizon and belongs to a class of metrics discussed in $[17,19,24]$, and one can apply the methods of these papers to obtain Virasoro algebra of surface deformations [32].

It is of interest to find further examples of metrics with trapping (apparent) horizons to which one can apply the above procedure. However it is not an easy job to find a null Killing vector for a general metric. A minor generalization of the above metrics is

$$
d s^{2}=-d t^{2}+a^{2}(t) b^{2}(r) d r^{2}+R^{2}(x) d \Omega^{2},
$$

where $a(t)$ and $b(r)$ are not fixed a priori. Transforming $b(r) d r=d \rho$ we obtain the metric with $b=1$ and transformed $\tilde{R}(x)$. Killing equations can be solved as in Sect.2, and $a(t)$ is determined from the equation $\ddot{a} a-\dot{a}^{2}=0$.

Demanding that at the horizon the Killing vector is null, we arrive at the equation

$$
\left(k_{1}+k_{2} \rho_{h}\right)^{2}=a^{2} H^{2}\left(k_{1} \rho_{h}+k_{2} \frac{\rho_{h}^{2}}{2}+\frac{k_{2}}{2 H^{2} a^{2}}+C\right)^{2},
$$

or, taking the square root of both parts of the relation with the sign plus,

$$
k_{1}\left(\frac{1}{H a}-\rho_{h}\right)=\frac{k_{2}}{2}\left(\frac{1}{H a}-\rho_{h}\right)^{2}+C .
$$

From this equation we obtain

$$
\rho_{h}=\frac{1}{H a}-\frac{k_{1}}{k_{2}} \pm \sqrt{\frac{k_{1}^{2}}{k_{2}^{2}}-C}=\frac{1}{H a}+\tilde{C} .
$$

To obtain a tractable form of equation for trapping horizon, $-\dot{\tilde{R}}^{2}+a^{-2} \tilde{R}^{\prime 2}=0$, we are forced again to assume a separable form of $\tilde{R}(x)$. The above form of $\rho_{h}$ is obtained by taking $\tilde{R}=a(t)(\rho-\tilde{C}) C^{\prime}$. Transforming back to variable $r$, we obtain $R(r, t)=a(t)\left(\int b(r) d r-\tilde{C}\right) C^{\prime}$.

\section{Appendix}

In this Appendix we explicitly verify some relations used in derivation of the central extension of the Virasoro algebra. We consider the case discussed in Sect. 2.1. 
Let us denote by $x$ variation of the radius of the trapping horizon $r_{h}=1 / \dot{a}(t): \quad r=r_{h}+x$. Under this variation the vectors $\chi^{a}$ and $\rho_{a}$ change as

$$
\begin{aligned}
\chi_{h}^{a}+\delta \chi^{a}= & \left(\left(k_{1}+\frac{k_{2}}{H a}\right)+k_{2} x ; \frac{1}{a}\left(k_{1}+\frac{k_{2}}{H a}\right)+x H\left(k_{1}+\frac{k_{2}}{H a}\right)\right) \\
\rho_{a, h}+\delta \rho_{a}= & \left(\left(k_{1}+\frac{k_{2}}{H a}\right)+x \frac{2 H a}{k_{1}}\left(k_{1}+\frac{k_{2}}{H a}\right)\left(k_{1}+\frac{k_{2}}{2 H a}\right) ;\right. \\
& \left.-a\left(k_{1}+\frac{k_{2}}{H a}\right)-x \frac{H a^{2}}{k_{1}}\left(k_{1}^{2}+\frac{3 k_{1} k_{2}}{H a}+\frac{k_{2}^{2}}{H^{2} a^{2}}\right)\right)
\end{aligned}
$$

It is explicitly verified that in the first order in $x \chi^{a} \rho_{a}=0$. From the expressions (A.1), (A.2) in the first order in $x$ we obtain

$$
\begin{aligned}
& \left(\chi_{h}+\delta \chi\right)^{2}=2 x k_{1} H a\left(k_{1}+\frac{k_{2}}{H a}\right), \\
& \left(\rho_{h}+\delta \rho\right)^{2}=-2 x k_{1} H a\left(k_{1}+\frac{k_{2}}{H a}\right) .
\end{aligned}
$$

Here $\chi_{h}^{2}$ and $\rho_{h}^{2}=0$. From the formulas (A.1)-(A.6) it follows that $|\chi / \rho| \rightarrow 1$ near horizon. It also follows that the vector

$$
k^{a}=\frac{1}{\chi^{2}}\left(\chi^{a}-\left|\frac{\xi}{\rho}\right| \rho^{a}\right)
$$

is finite on the horizon.

From (A.1), (A.2) we obtain

$$
\chi_{r} \chi_{t}-\chi_{t} \chi_{r}=2 x k_{1} H a^{2}\left(k_{1}+\frac{k_{2}}{H a}\right)
$$

Calculating

$$
\nabla_{r} \chi_{t}=-\nabla_{t} \chi_{r}=H a k_{1},
$$

from the formulas (A.6), (A.7) and (A.4) we have an important relation

$$
\nabla_{a} \chi_{b}=\frac{\kappa}{\chi^{2}}\left(\chi_{a} \rho_{b}-\chi_{b} \rho_{a}\right) .
$$

In the same way, calculating $\chi_{a} \chi_{b}-\rho_{a} \rho_{b}$ (as an example we present result for $a, b=r, t$ )

$$
\chi_{r} \chi_{t}-\rho_{r} \rho_{t}=2 x a^{2} H\left(k_{1}+\frac{k_{2}}{H a}\right)^{3}
$$

and

$$
\nabla_{r} \chi_{t}=\nabla_{t} \chi_{r}=\frac{H a}{k_{1}}\left(k_{1}+\frac{k_{2}}{H a}\right)^{2}
$$

we obtain

$$
\nabla_{a} \rho_{b}=\frac{\kappa}{\chi^{2}}\left(\left(\chi_{a} \rho_{b}-\chi_{b} \rho_{a}\right) .\right.
$$

Relations (A.8) and (A.11) are the main building blocks leading to the formula (59) It follows also that the surface element

$$
\mu_{a b}=-\left|\frac{\chi}{\rho}\right|\left(\chi_{a} \rho_{b}-\chi_{b} \rho_{a}\right)
$$

is finite at the horizon and $\mu_{r t}=a(t)$. 


\section{References}

[1] J.D. Bekenstein, Phys. Rev. D7, 2333 (1973).

[2] S.W. Hawking, Nature 248, 30 (1974).

[3] G.W. Gibbons and S.W. Hawking, Phys. Rev. D15, 2738 (1977).

[4] S.A. Hayward, Class. Quant. Grav. 15, 3147 (1988).

[5] S. A. Hayward, S. Mukohyama and M.C. Ashworth, Phys. Lett. A256, 347 (1999).

[6] P.C.W. Davies, Class. Quant. Grav. 5, 1349 (1988).

[7] R.-G. Cai, L.-M. Cao, Nucl. Phys. B785, 135 (2007).

[8] V. Faraoni, Phys. Rev. D84, 024003 (2011).

[9] A. Ashtekar and B. Krishnan, Living Rev. Relat. 7, 10 (2004).

[10] A.B. Nielsen, Gen. Rel. Grav. 41, 1539 (2009).

[11] A.B. Nielsen and D.H. Yeom, Int. J. Mod. Phys. A24, 5261 (2009).

[12] A.B. Nielsen and M. Visser, Class. Quant. Grav. 23, 4637 (2006).

[13] A. Strominger, JHEP 9802, 009 (1998); D. Birmingam, I. Sachs, S. Sen, Phys. Lett., B 424, 275 (1998).

[14] J. D. Brown and M. Henneaux, Commun. Math. Phys. 104, 207 (1986).

[15] G. Barnich and F. Brandt, Nucl. Phys. B 633, 3 (2002); G. Barnich and G. Compere, J. Math. Phys. 49, 042901 (2008).

[16] M. Guica, T. Hartman, W. Song and A. Strominger, Phys. Rev. D 80, 124008 (2009).

[17] S. Carlip, Class. Quant. Grav.16, 3327 (1999); S. Carlip, Phys. Rev. Lett. 82, 2828 (1999).

[18] Mu-in Park, Nucl. Phys. B 634, 339 (2002).

[19] S. Carlip, Extremal and nonextremal Kerr/CFT correspondences arXiv:1101.5136.

[20] G. Kang, J.-i. Koga and M.-I. Park, Phys.Rev. D 70, 024005 (2004).

[21] S. Carlip, Effective Conformal Descriptions of Black Hole Entropy: A Review arXiv:1207.1488.

[22] M. Cvitan, S. Pallua and P. Prester, Phys.Rev. D 70, 084043 (2004).

[23] S. Carlip, Class. Quant. Grav. 15, 3609 (1998).

[24] B. R. Majhi and T. Padmanabhan, Phys. Rev. D 85, 084040 (2012); ibid D 86 (2012) 101501(R);

B. R. Majhi, Adv. High Energy Phys. 2013, 386342 (2013).

[25] R. M. Wald, Phys. Rev. D 48, 3427 (1993); ibid V. Iyer and R. M. Wald, D 50, 846 (1994). 
[26] X.-N. Wu and Y. Tian, Phys. Rev. D 80, 024014 (2009); C.-Y. Zhang, Y. Tian, X.-N. Wu, S.-J. Zhang, Entropy of isolated horizon from surface term of gravitational action, arXiv:1403.7352.

[27] H. Kodama, Progr. Theor. Phys. 63, 1217 (1980).

[28] S. A. Hayward, Phys. Rev. D 49, 6467 (1994); ibid D 53, 1938 (1996).

[29] S. A. Hayward, Relativistic thermodynamics., arXiv:gr-qc/9803007.

[30] R. Di Criscienzo, S.A. Hayward, M. Nadalini, L. Vanzo and S. Zerbini, Class. Quant. Grav. 27, 015006 (2010).

[31] T. Padmanabhan, Class. Quant. Grav. 19, 5387 (2002); Phys. Rept. 406, 49 (2005).

[32] F.-L. Lin and Y.-S. Wu, Phys. Lett. B 453, 222 (1999). 